\title{
Applications and limitations of electron correlation microscopy to study relaxation dynamics in supercooled liquids
}

\author{
Pei Zhang ${ }^{1}$, Li He ${ }^{1}$, Matthew F. Besser ${ }^{2}, Z_{\text {Ze }}$ Liu $^{3}$, Jan Schroers ${ }^{3}$, Matthew J. Kramer ${ }^{2}$, Paul M. Voyles ${ }^{1 *}$ \\ ${ }^{1}$ Department of Materials Science and Engineering, University of Wisconsin-Madison, Madison, \\ Wisconsin 53706, USA. \\ ${ }^{2}$ Materials Science and Engineering, Ames Laboratory, Iowa State University, Ames, Iowa 50011, USA. \\ ${ }^{3}$ Department of Mechanical Engineering and Materials Science, Yale University, New Haven, \\ Connecticut 06511, USA.
}

Corresponding author: Paul M. Voyles Email address: paul.voyles@wisc.edu

\begin{abstract}
Electron correlation microscopy (ECM) is a way to measure structural relaxation times, $\tau$, of liquids with nanometer-scale spatial resolution using coherent electron scattering equivalent of photon correlation spectroscopy. We have applied ECM with a $3.5 \mathrm{~nm}$ diameter probe to $\mathrm{Pt}_{57.5} \mathrm{Cu}_{14.7} \mathrm{Ni}_{5.3} \mathrm{P}_{22.5}$ amorphous nanorods and $\mathrm{Pd}_{40} \mathrm{Ni}_{40} \mathrm{P}_{20}$ bulk metallic glass (BMG) heated inside the STEM into the supercooled liquid region. These data demonstrate that the ECM technique is limited by the characteristics of the time series, which must be at least $40 \tau$ to obtain a well-converged correlation function $g_{2}(t)$, and the time per frame, which must be less than $0.1 \tau$ to obtain sufficient sampling. A high-speed direct electron camera enables fast acquisition and affords reliable $g_{2}(t)$ data even with low signal per frame.
\end{abstract}

Keywords: electron correlation microscopy; supercooled liquid; structural relaxation time; metallic glass; in-situ heating 


\section{Introduction}

Electron correlation microscopy uses time-resolved coherent electron nanodiffraction to measure atomic dynamics in materials [1]. It is modeled and named after photon correlation spectroscopy (PCS) [2], a similar technique using photons. Coherent scattering with either photons or electrons gives rise to speckle patterns, in which each speckle corresponds to a volume of the sample with sufficient internal order to create constructive interference of the scattered waves. The time of which the speckle intensity persists then corresponds to the time over which that particular structure persists. The persistence time can be measured statistically from the time autocorrelation function,

$$
g_{2}(t)=\frac{<I\left(t^{\prime}\right) I\left(t^{\prime}+t\right)>}{<I\left(t^{\prime}\right)>^{2}},
$$

where $t^{\prime}$ is the time of a frame in the diffraction time series, $t$ is delay time after $t^{\prime}$, and $\langle>$ denotes average over all $t^{\prime}$.

The systems accessible to PCS and related techniques depend on the coherence length of the illumination and the wavelength of the radiation. PCS with optical lasers can produce large transverse coherence lengths, but the wavelength limits applications to systems like colloids with micron scale or larger particles. Coherent x-ray beams from synchrotron sources have enabled X-ray PCS (XPCS) [3], first with soft x-rays capable of studying smaller colloids and polymers [4,5], then with steadily harder X-rays that are now capable of studying atomic-scale motions [68]. In addition to studying particle dynamics, XPCS has been used to study atomic diffusion in alloys [9], and fluctuating ferromagnetic and ferroelectric domain structures [10,11].

The advantages of ECM over XPCS are the typical advantages of electron scattering over X-rays: high-quality lenses make nanometer-scale coherent probes easily accessible on any modern field-emission gun scanning transmission electron microscope (STEM) [12,13] and large elastic scattering factors create significant diffraction even from small volumes. The comparatively large signal may make it easier to measure fast dynamics from small structures in ECM than in XPCS. The disadvantages are also typical of electron microscopy: ECM requires thin samples without superficial oxidation, and maintaining sample and instrument stability over long experiments is a challenge. As a result, very slow dynamics are likely better measured using XPCS.

Our first experiments with ECM have focused on the atomic-scale dynamics in bulk metallic glass forming alloys heated above the glass transition temperature $T_{g}$ and into the supercooled liquid [1]. Glassy dynamics occur via two general processes, called the $\alpha$ and $\beta$ relaxations [14]. The $\alpha$ relaxation is associated with collective structural rearrangements in the liquid involving multiple atoms changing nearest neighbors. The $\beta$ relaxation involves singleatom hopping or diffusion. As the temperature falls toward $T_{g}$, the $\alpha$ relaxation time $\tau_{\alpha}$ diverges, but the $\beta$ relaxation time $\tau_{\beta}$ does not. (This is a somewhat idealized picture. In some real glasses, the $\mathrm{a}$ and $\mathrm{b}$ relaxations are not easily distinguished from one another [15]). $\tau_{\alpha}$ and $\tau_{\beta}$ are often measured by applying a time-varying stimulus to a material and measuring the out-of-phase response as a function of frequency. Different stimuli lead to different techniques, including dielectric relaxation [16], dynamic mechanical analysis [17-19], or modulated differential scanning calorimetry [20]. 
If $g_{2}(t)$ from ECM or XPCS is averaged over many speckles and many speckle liftetimes, it can be modeled by [21]

$$
g_{2}(t)=1+A \exp \left[-2\left(\frac{t}{\tau}\right)^{\beta}\right]
$$

where $\tau$ is the structural relaxation time, usually identified with $\tau_{\alpha}$ for supercooled liquids with a distinct $\tau_{\alpha}$ and $\tau_{\beta}$. $\quad \beta$ is a stretching exponent with the same physical meaning as $\beta$ in the Kohlrausch-Williams-Watt equation often used to describe relaxation behavior in glasses: $\beta=1$ is exponential decay, and $\beta<1$ corresponds to the superposition of several relaxation processes with different characteristic times or rates. $A$ depends on the contrast of the speckles above the incoherent background and noise which in turn depends on the coherence of the illumination and other experimental parameters. The value of ECM is the ability to measure $g_{2}(t)$ and thus $\tau$ with nanometer spatial resolution.

In this paper, we determine the experimental requirements for ECM technique through the measurement of the relaxation time in $\mathrm{Pd}_{40} \mathrm{Ni}_{40} \mathrm{P}_{20}$ bulk metallic glass and $\mathrm{Pt}_{57.5} \mathrm{Cu}_{14.7} \mathrm{Ni}_{5.3} \mathrm{P}_{22.5}$ metallic glass nanorods. We report first results from ECM experiments using a fast direct electron detection camera to improve the time resolution. Despite the low probe current required to achieve good coherence and the resulting low number of electrons detected in each diffraction pattern, the $g_{2}(t)$ data obtained from tens of thousands of patterns are low noise and an excellent match to Equation (2). We also discuss the time resolution and total time series length required for ECM experiments to obtain reliable $g_{2}(t)$ results.

\section{Experimental Methods}

\section{Materials preparation}

$\mathrm{Pt}_{57.5} \mathrm{Cu}_{14.7} \mathrm{Ni}_{5.3} \mathrm{P}_{22.5}$ glassy nanorods with diameter $\sim 35 \mathrm{~nm}$ were synthesized by the nanomoulding method [22] which is based on thermoplastic forming of the BMG in its supercooled liquid region. In order to minimize the residual salts and anodized aluminium oxide from nanomoulding, we rinsed the plate with the rods attached in distilled water and isopropyl alcohol at least three times. Then the plate was immersed in methanol and the nanorods were released by by sonication for 15 20 mins. A micro pipet was used to drop $1.5-1.8 \mu \mathrm{L}$ of methanol containing nanorods onto a microhotplate in situ TEM heater chip from DENSSolutions [23]. Some of the nanorods attached to the $\mathrm{SiN}_{\mathrm{x}}$ membrane of chip window after the evaporation of methanol. We repeated the drop process several times to increase the density of nanorods on the chip. To remove the fairly severe contamination induced by this preparation method, the sample holder with the chip was plasma cleaned at $20 \mathrm{psi} \mathrm{Ar}+\mathrm{O}_{2}$ mixture for $12 \sim 15$ mins before ECM measurements.

A $\mathrm{Pd}_{40} \mathrm{Ni}_{40} \mathrm{P}_{20}$ metallic glass ingot $1 \mathrm{~mm}$ diameter was synthesized by injection casting. The details for fabrication are described in detail elsewhere [1]. TEM specimens were prepared by focused ion beam (FIB) lift-out in a Zeiss Auriga cross beam FIB. First, the ion beam and the electron beam were used to deposit a $2 \mu \mathrm{m}$ thick Pt protection layer, then a $\mathrm{Pd}_{40} \mathrm{Ni}_{40} \mathrm{P}_{20}$ lamella around $15 \mu \mathrm{m} \times 10 \mu \mathrm{m} \times 2.5 \mu \mathrm{m}$ in size was extracted and attached to a copper grid with $\mathrm{Pt}$ deposition. The lamella was thinned to $\sim 300 \mathrm{~nm}$ thick with $30 \mathrm{kV}, 80 \mathrm{pA} \mathrm{Ga}$ ion, then the current was reduced to $20 \mathrm{pA}$ for milling until the sample became electron transparent to $7 \mathrm{kV}$ 
SEM imaging. Then a $5 \mathrm{kV}, 20 \mathrm{pA}$ ion beam was used for milling until sample was electron transparent to $3 \mathrm{kV}$ SEM imaging. Finally, a $2 \mathrm{kv}, 100 \mathrm{pA}$ ion beam was used to remove surface damage. The stage was tilted by around 2 degrees off the Ga emission direction during thinning. Next sample was transferred and attached over an empty window in a microhotplate heating chip window at a $13^{\circ}$ incline angle to the chip surface using Pt deposition. Then $2 \mathrm{kv}, 100 \mathrm{pA} \mathrm{Ga}+$ ion was used to remove Pt redeposition on sample surface. These samples were plasma cleaned at 20 psi $\mathrm{Ar}+\mathrm{O}_{2}$ mixture for $\sim 30 \mathrm{~s}$ before ECM measurements.

\section{Electron Correlation Microscopy Experiments}

Low-speed ECM on the $\mathrm{Pd}_{40} \mathrm{Ni}_{40} \mathrm{P}_{20}$ sample was performed in the University of Wisconsin-Madison FEI Titan STEM with probe aberration corrector at $200 \mathrm{kV}$ in energy filtered "microprobe" STEM ( $\mu$ P-EFSTEM) mode. A camera length of $512 \mathrm{~mm}$ was used and the semi-convergence angle was adjusted to obtain probe with $3.5 \mathrm{~nm}$ diameter. The probe current was $1.6 \mathrm{pA}$. A Gatan BF_DF detector mounted on the $2.5 \mathrm{~mm}$ GIF entrance aperture was used to collect STEM images and Gatan US 1000 CCD camera inside a GIF 865ER energy filter was used to collect nanodiffraction patterns. The diffraction patterns were zero-loss energy filtered with a slit width of $10 \mathrm{eV}$. At binning factor 8 (256 by 256 pixel images) the readout time for the US1000 camera is $0.07 \mathrm{~s}$. For lower temperatures from $328{ }^{\circ} \mathrm{C}$ and $332{ }^{\circ} \mathrm{C}$, the exposure time $0.2 \mathrm{~s}$ for a total time per frame of $0.27 \mathrm{~s}$. For temperatures from $336{ }^{\circ} \mathrm{C}$ to $352{ }^{\circ} \mathrm{C}$, the exposure time was $0.1 \mathrm{~s}$ for a total time per frame of $0.17 \mathrm{~s}$. Every series consists of $3000 \sim$ 4000 frames. The total electron dose on sample is $7.3 \times 10^{8} \sim 8.3 \times 10^{8} \mathrm{e}^{-} / \mathrm{nm}^{2}$.

High-speed ECM experiments on the $\mathrm{Pt}_{57.5} \mathrm{Cu}_{14.7} \mathrm{Ni}_{5.3} \mathrm{P}_{22.5}$ nanorods were obtained on the Brookhaven National Lab image-corrected Titan ETEM at $300 \mathrm{kV}$, a probe size of $3.5 \mathrm{~nm}$, and a probe current of $10.5 \mathrm{pA}$, using a Gatan K2-IS direct electron detection camera, without energy filtering. The K2-IS acquires 1920 by 1856 pixel images at $2.5 \mathrm{~ms}$ total frame time ( 400 frames per second) and almost zero readout overhead time. Each time series of nanodiffraction patterns was two minutes long, so it contains 48,000 frames and occupies $\sim 2.5 \mathrm{~TB}$ of data storage uncompressed. For each measurement, the total electron dose on sample is $\sim 8.2 \times 10^{8} \mathrm{e}^{-} / \mathrm{nm}^{2}$. The first step in the analysis was to crop each image to smaller size to include just the first amorphous ring which will be used to calculate the autocorrelation function. Since ECM does not require this high pixel count, the image was then binned by 10. Even after binning, each speckle covers 3-4 pixels. Large volume data analysis was performed using data storage and computing provided by the Brookhaven Center for Functional Nanomaterials and RHIC / ATLAS data center.

Samples were heated inside both microscopes using a DENSSolutions SH30 single-tilt heating holder, which provides temperature stability of $\pm 0.03{ }^{\circ} \mathrm{C}$ and sample drift rates comparable to room temperature operation of the same microscope. The usable temperature range for these experiments is from $T_{g}$ to the crystallization temperature $T_{x}$, which is $300{ }^{\circ} \mathrm{C}$ to $374{ }^{\circ} \mathrm{C}$ for $\mathrm{Pd}_{40} \mathrm{Ni}_{40} \mathrm{P}_{20}$ and $230{ }^{\circ} \mathrm{C}$ to $265{ }^{\circ} \mathrm{C}$ for $\mathrm{Pt}_{57.5} \mathrm{Cu}_{14.7} \mathrm{Ni}_{5.3} \mathrm{P}_{22.5}$. For both samples, initial heating from room temperature to above $T_{g}$ was $20^{\circ} \mathrm{C} / \mathrm{min}$. Then at each test temperature, the sample was held isothermally for 2 mins before data collection.

$g_{2}(t)$ was calculated from the diffraction data as 


$$
g_{2}(p)=\frac{(N-p) \sum_{i=0}^{N-p-1} I(i) I(i+p)}{\left[\sum_{i=0}^{N-p-1} I(i)\right]\left[\sum_{i=0}^{N-p-1} I(i+p)\right]},
$$

where $\mathrm{N}$ is the total number of frames in the diffraction time series, and $p$ is the frame / time index. $g_{2}(t)$ was calculated for every pixel $\left(k_{x}, k_{y}\right)$ in the diffraction pattern except the part covered by beam stop, then averaged over the ring of pixels at constant $k=\sqrt{k_{x}^{2}+k_{y}^{2}}$. All of the data reported here are for the first diffuse ring in the nanodiffraction pattern, which is $k \sim$ $0.45 \AA^{-1}$ for $\mathrm{Pd}_{40} \mathrm{Ni}_{40} \mathrm{P}_{20}$ and $0.44 \AA^{-1}$ for $\mathrm{Pt}_{57.5} \mathrm{Cu}_{14.7} \mathrm{Ni}_{5.3} \mathrm{P}_{22.5}$. The averaged $g_{2}(t)$ was resampled logarithmically in time, then fit to Equation (2) using standard non-linear least-squares fitting.

\section{Results and discussion}

Figure 1(a) shows an example of single nanodiffraction pattern from a $\mathrm{Pt}_{57.5} \mathrm{Cu}_{14.7} \mathrm{Ni}_{5.3} \mathrm{P}_{22.5}$ nanorod at $250{ }^{\circ} \mathrm{C}$ acquired on the $\mathrm{K} 2$-IS camera after cropping and binning. Figure 1(b) is the normalized, resampled $g_{2}(t)$ extracted from the time series of nanodiffraction patterns like Figure 1(a). Although only a few $\mathrm{e}^{-}$are recorded per frame, the aggregate statistics from the series of 48,000 frames are low noise and an excellent fit to Equation (2).

a

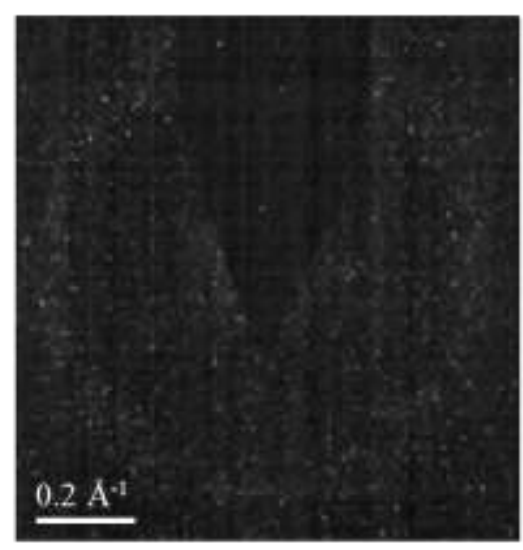

b

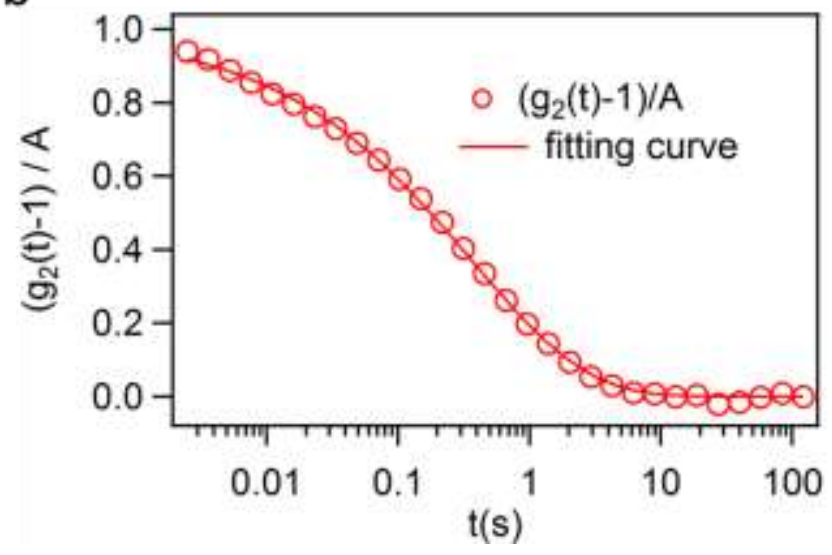

Figure 1. (a) A typical nano beam electron diffraction pattern of $\mathrm{Pt}_{57.5} \mathrm{Cu}_{14.7} \mathrm{Ni}_{5.3} \mathrm{P}_{22.5}$ nanowire at $250{ }^{\circ} \mathrm{C}$ recorded by $\mathrm{K} 2$ detector with speed of $2.5 \mathrm{~ms}$ per frame. (b) The normalized time autocorrelation function $g_{2}(t)$ and the fitting curve based on Equation (2).

Figure 2(a) shows the change of structural relaxation time $\tau$ with temperature in the supercooled $\mathrm{Pt}_{57.5} \mathrm{Cu}_{14.7} \mathrm{Ni}_{5.3} \mathrm{P}_{22.5}$ nanowire measured with the K2-IS camera. The time series of nanodiffraction pattern was collected near the nanowire surfaces and the autocorrelation function was calculated from $k \sim 0.44 \AA^{-1}$. $\tau$ continually decreases with temperature increasing from $242{ }^{\circ} \mathrm{C}$ to $258{ }^{\circ} \mathrm{C}\left(T_{g}+12{ }^{\circ} \mathrm{C}\right.$ to $\left.T_{g}+28{ }^{\circ} \mathrm{C}\right)$. Since no report is available in the literature for the $\tau_{\alpha}$ of bulk $\mathrm{Pt}_{57.5} \mathrm{Cu}_{14.7} \mathrm{Ni}_{5.3} \mathrm{P}_{22.5}$, there is no direct comparison between nanowire and bulk at the same composition. However, compared with the typical $\tau$ measured from BMGs [20,24-26], the $\tau$ is much shorter, indicating a higher mobility and faster dynamics in the nanowire. The stretching exponent $\beta$ in Figure 2(b) decreases with higher temperatures, indicating a wider 
distribution of relaxation time and more spatial heterogeneity of atomic dynamics when temperature approaching $T_{x}$.

Figure 2 also shows ECM data from the same sample acquired at a slower frame rate with a CCD camera. From $242{ }^{\circ} \mathrm{C}$ to $250{ }^{\circ} \mathrm{C}$, when the relaxation time is relatively long, the $\mathrm{K} 2$ and $\mathrm{CCD}$ relaxation time results agree within experimental uncertainty. At higher temperature and shorter $\tau$, the results diverge due to insufficient temporal resolution on the CCD. The requirements for temporal resolution and the length of the time series are discussed below.
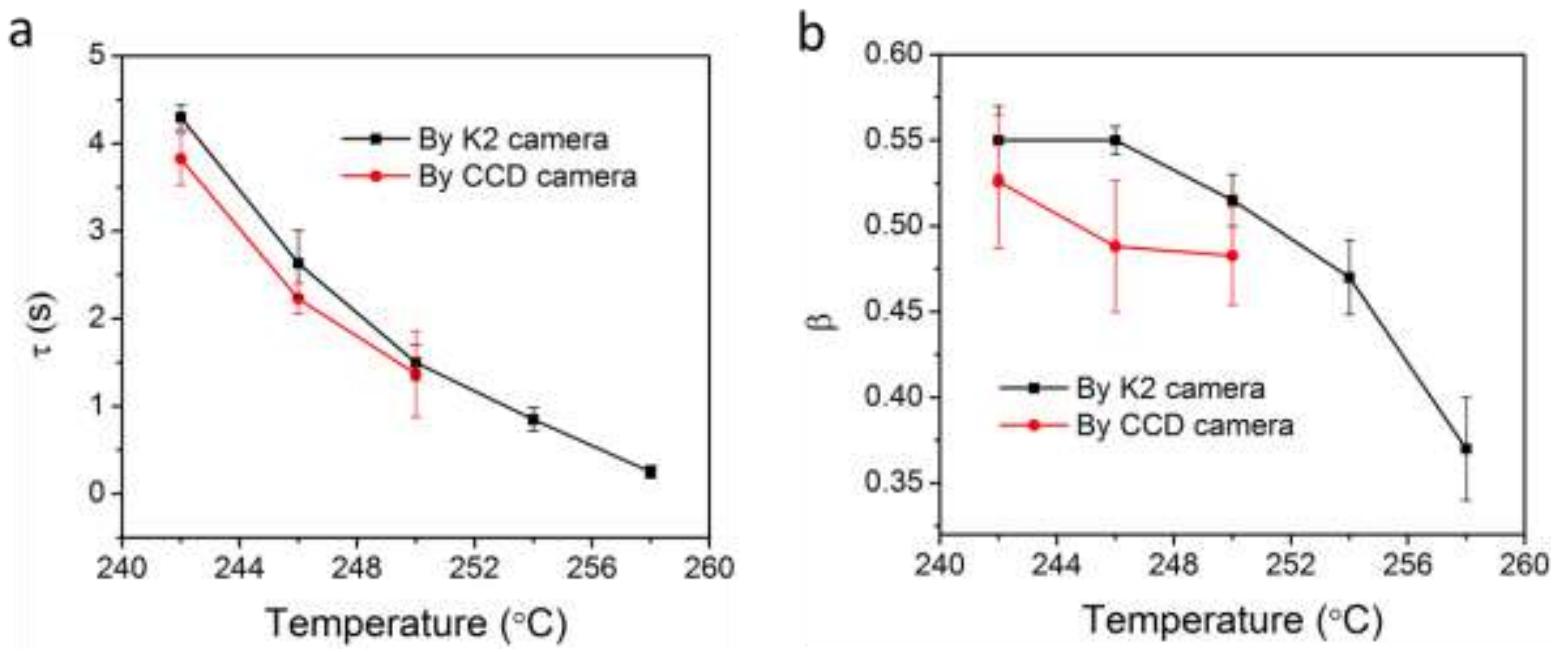

Figure 2. (a) Structural relaxation time $\tau$ and (b) stretching exponent $\beta$ in the supercooled liquid region of $\mathrm{Pt}_{57.5} \mathrm{Cu}_{14.7} \mathrm{Ni}_{5.3} \mathrm{P}_{22.5}$ nanowire from both the $\mathrm{K} 2$ camera and a CCD camera. The error is the standard deviation of the mean from three different measurements.

Figure 3(a) and (b) shows the structural relaxation time $\tau$ and stretching exponent $\beta$ in the supercooled liquid region of $\mathrm{Pd}_{40} \mathrm{Ni}_{40} \mathrm{P}_{20} \mathrm{BMG}$ measured using the CCD camera. The time autocorrelation function $g_{2}(t)$ is derived from $k \sim 0.45 \AA^{-1}$. $\tau$ shows a continous decreasing with temperature increasing from $328{ }^{\circ} \mathrm{C}$ to $348{ }^{\circ} \mathrm{C}\left(T_{g}+28{ }^{\circ} \mathrm{C}\right.$ to $\left.T_{g}+48{ }^{\circ} \mathrm{C}\right)$. As the temperature approaches crystallization temperature $T_{x}$, there is an obvious increase of relaxation time, caused by the growth of nuclei and crystal from some pre-existing ordered structures possibly induced during sample preparation by FIB. Compared with the previous ECM measurement in $\mathrm{Pd}_{40} \mathrm{Ni}_{40} \mathrm{P}_{20}$ BMG by Li [1], a similar increasing of $\tau$ arinsing from crystallization was also reported when the temperature is above $340{ }^{\circ} \mathrm{C}$, but from temperature $328{ }^{\circ} \mathrm{C}$ to $340{ }^{\circ} \mathrm{C}, \tau$ measured here is generally larger (e.g. $\sim 25 \mathrm{~s}$ at $328{ }^{\circ} \mathrm{C}$ here compared with $\sim 11 \mathrm{~s}$ at $325{ }^{\circ} \mathrm{C}$ in Li's report), that is because a longer trajectory length was applied here to ensure the convergence of $g_{2}(t)$ and capture the whole relaxation behavior, which will be discussed in detail in Figure 5. Accoring to the report by Wilde [20], $\tau$ measured at $340{ }^{\circ} \mathrm{C}$ from ECM experiement is very close to that obtained from modulated differential scanning calorimetry (MDSC). The stretching exponent $\beta$, an indicater of spatial heterogeniety of relaxation dymamics, is typically smaller than 1 in metallic glass systems. Figure 3(b) shows that the $\beta$ is quite scattered with no 
obvious tendency with temperature change. The $\beta$ averaged from the whole measured temperature region is around 0.44 , which is comparable to other measurements $[1,27,28]$.
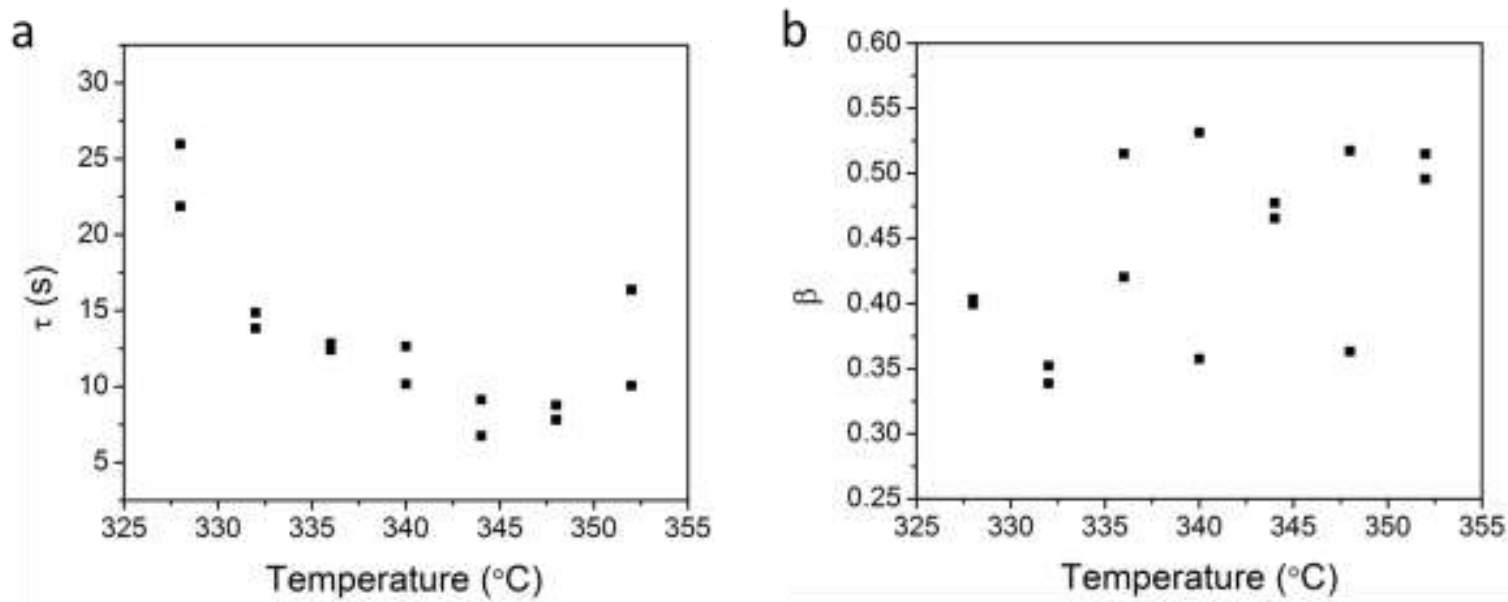

Figure 3. (a) Structural relaxation time $\tau$ and (b) stretching exponent $\beta$ in the supercooled liquid region of $\mathrm{Pd}_{40} \mathrm{Ni}_{40} \mathrm{P}_{20} \mathrm{BMG}$.

Figure 4 shows that the $A$ parameter in Equation (2) is effected by the probe coherence and probe current. The probe coherence can be varied by changing the source demagnification using the excitation of the first condenser lens. This control is called "spot size" on FEI microscopes. Yi and Voyles have shown that varying spot size systematically changes the coherence length of the illumination field in the condenser aperture plane [13]. Figure 4 shows that $A$, measured from $\mathrm{Pd}_{40} \mathrm{Ni}_{40} \mathrm{P}_{20}$ metallic glass at $320^{\circ} \mathrm{C}$, increases with increasing coherence length and FEI spot number. Increasing spot number and coherence also decreases the probe current, showing quantitatively that ECM delivers higher signal at lower probe current. We cannot currently configure the optics of the Titan STEM to vary the coherence without simultaneously varying either the probe current or the probe size. 


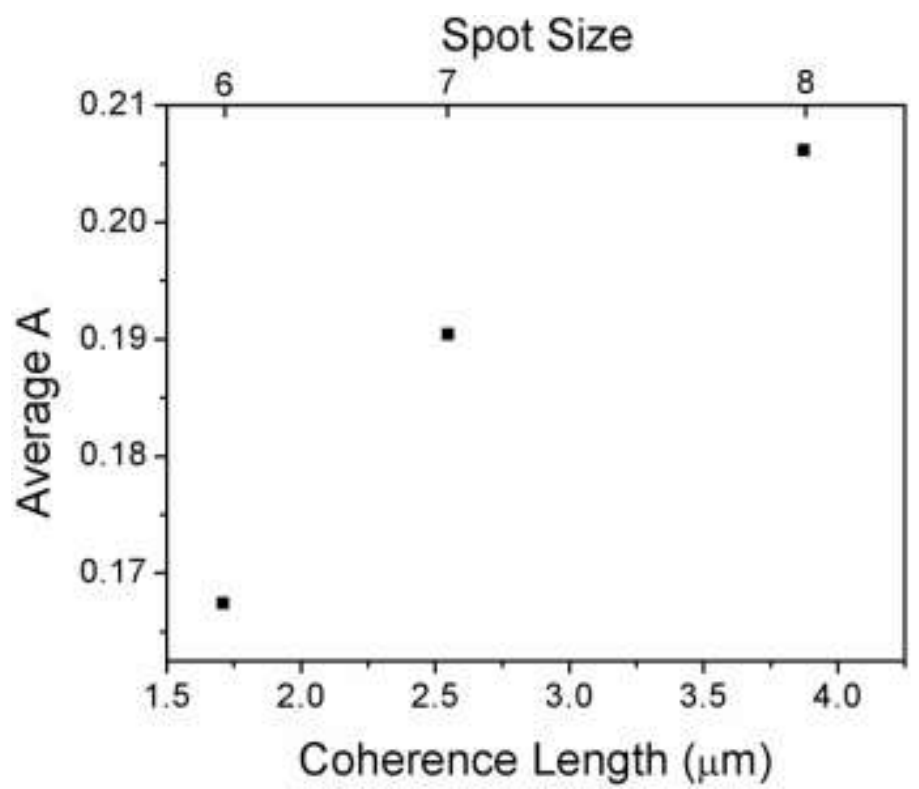

Figure 4. A determined by fitting to Equation (2) as a function of microscope spot size and coherence length in the $\mathrm{C} 2$ aperture plane, which is used to change the probe partial coherence. Data are from $\mathrm{Pd}_{40} \mathrm{Ni}_{40} \mathrm{P}_{20}$ measured at $320{ }^{\circ} \mathrm{C}$.

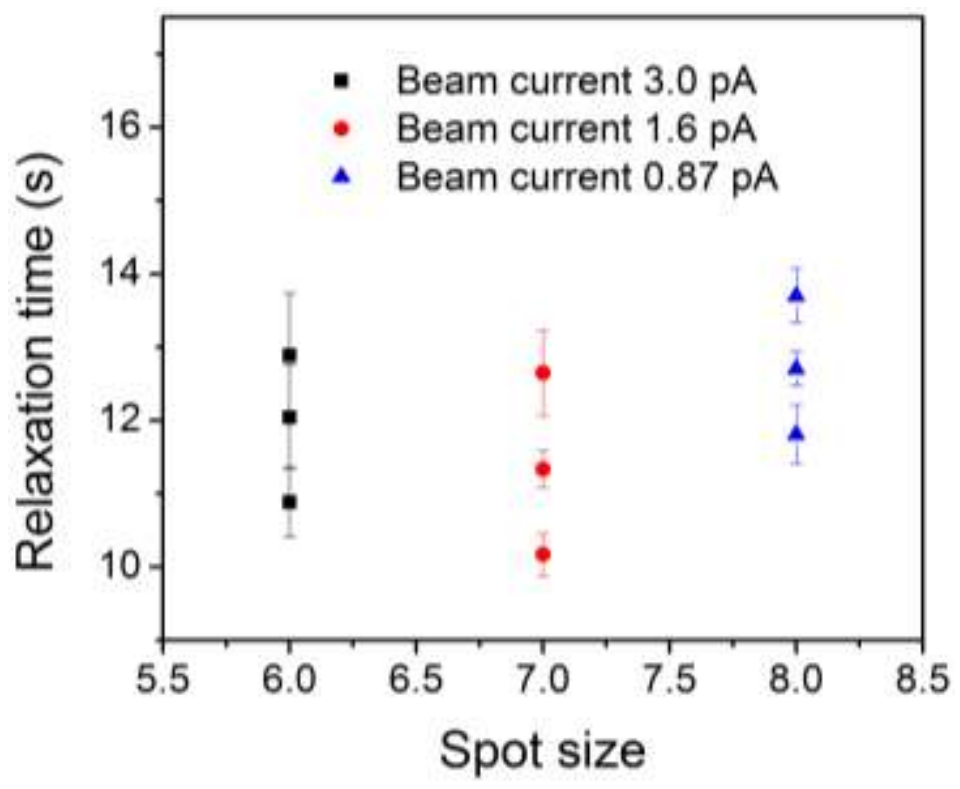

Figure 5. Structural relaxation time measured with different spot number and beam current. All the data are from $\mathrm{Pd}_{40} \mathrm{Ni}_{40} \mathrm{P}_{20} \mathrm{BMG}$ acquired at $340{ }^{\circ} \mathrm{C}$ with probe size $3.5 \mathrm{~nm}$. The error bar is from the uncertainty of the fitting with $g_{2}(t)$ correlation function.

Figure 5 shows the relaxation time as a function of spot size. For each spot size, the relaxation time is determined from three independent time series. The error bars represent the uncertainty in $\tau$ estimated from the non-linear fitting algorithm. These results show that $\tau$ as a 
function of beam current is within the experimental uncertainty of repeated measurements. However, the uncertainty from the fit gets larger at smaller spot number because the decrease in $A$ shown in Figure 4 means there is a smaller variation in $g_{2}(t)$.

The length of the time series is one significant factor that limits the application of the ECM technique. The time series must be much longer than $\tau$ for the $g_{2}(t)$ correlation function to converge, enabling reliable measurement of $\tau$. Figure 6 shows that for both materials studied here (bulk $\mathrm{Pd}_{40} \mathrm{Ni}_{40} \mathrm{P}_{20}$ at $332{ }^{\circ} \mathrm{C}$ and $\mathrm{Pt}_{57.5} \mathrm{Cu}_{14.7} \mathrm{Ni}_{5.3} \mathrm{P}_{22.5}$ nanorods at $254{ }^{\circ} \mathrm{C}$ ) a time series of at least $40 \tau$ is required to obtain reliable results. For bulk $\mathrm{Pd}_{40} \mathrm{Ni}_{40} \mathrm{P}_{20}$, the calculated $\tau$ becomes $23 \%$ shorter with the data length of $20 \tau$ and $41 \%$ shorter with $10 \tau$. A more dramatic decrease of calculated $\tau$ can be observed in $\mathrm{Pt}_{57.5} \mathrm{Cu}_{14.7} \mathrm{Ni}_{5.3} \mathrm{P}_{22.5}$ nanowire when the data length decreases to $20 \tau$ and $10 \tau$. This observation is consistent with other similar convergence tests for time autocorrelation functions calculated from time series [29]. When the time series is too short, $g_{2}(t)$ fails to reach a limiting value of 1 by the maximum $t$ (not shown). Thus, failure of $g_{2}(t)$ to converge to 1 is a practical indication of a time series that is too short.

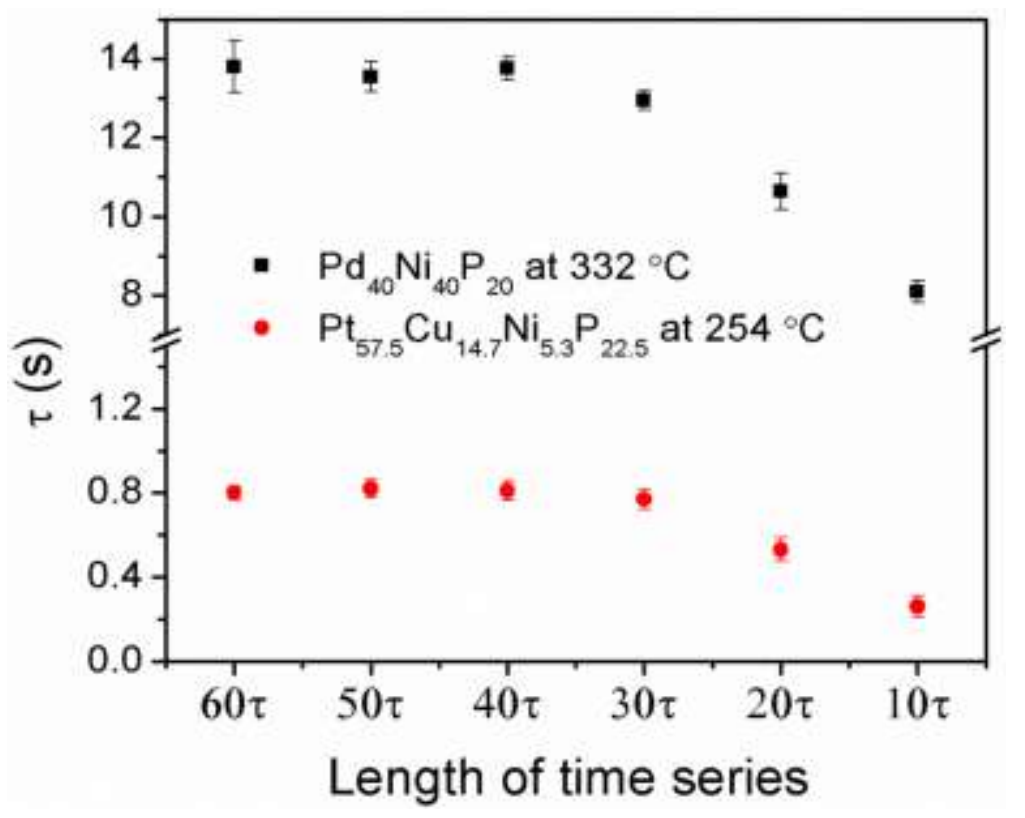

Figure 6. The effect of the length of time series on the structural relaxation time $\tau$ for both $\mathrm{Pd}_{40} \mathrm{Ni}_{40} \mathrm{P}_{20} \mathrm{BMG}$ and $\mathrm{Pt}_{57.5} \mathrm{Cu}_{14.7} \mathrm{Ni}_{5.3} \mathrm{P}_{22.5}$ nanowire.

This limitation can explain why our ECM measurements are comparable with results from macroscopic techniques at higher temperature when $\tau$ is short, but diverge at low temperatures for bulk $\mathrm{Pd}_{40} \mathrm{Ni}_{40} \mathrm{P}_{20}$ (not shown in figure 3). When the temperature is approaching $T_{g}$, the structural relaxation time is at the scale of several hundreds of seconds $[18,20,24]$. For ECM measurement, the required time series length would be hours to get converged data at low temperatures. Over such long times, the speckle lifetime will be dominated by drift of the sample, not intrinsic structural changes. Sample drift in the data in Figure 3 was $\sim 0.1 \mathrm{~nm} / \mathrm{min}$ and the entire drift for the longest time series $(1080 \mathrm{~s})$ was $\sim 1.8 \mathrm{~nm}$, which is in tolerance with $3.5 \mathrm{~nm}$ spatial resolution. 


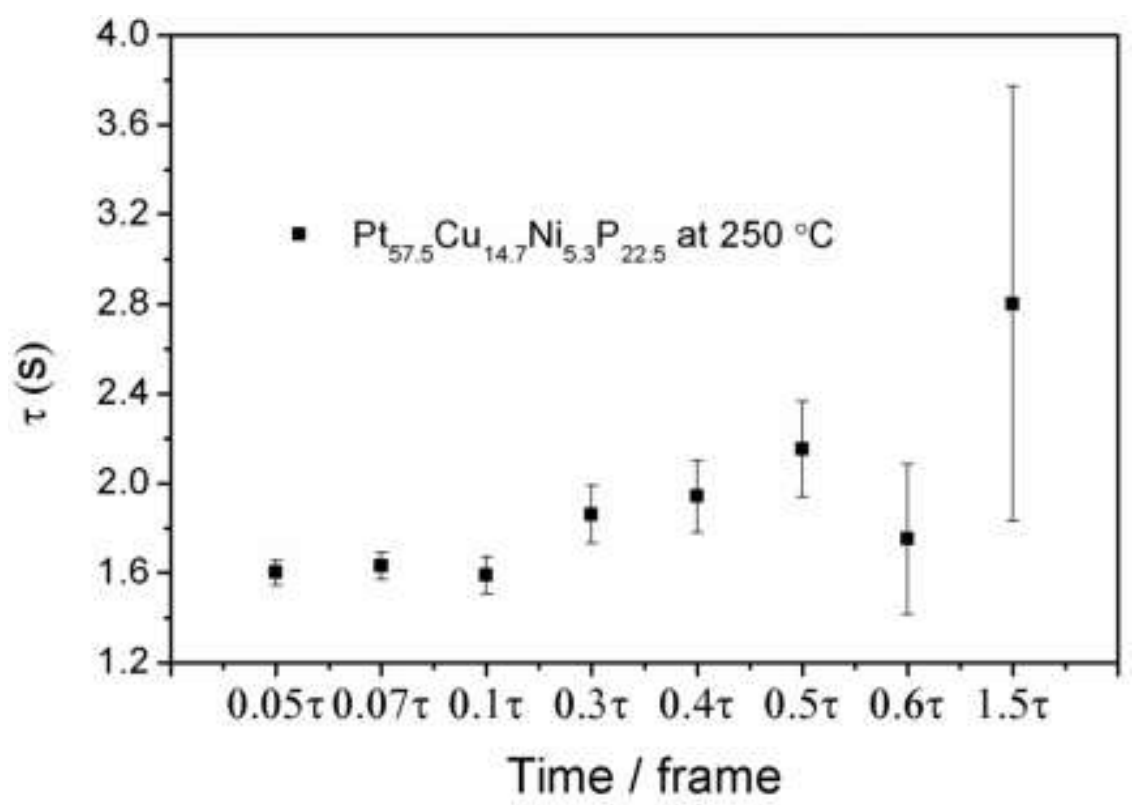

Figure 7. The influence of time per frame on structural relaxation time $\tau$ measured from $\mathrm{Pt}_{57.5} \mathrm{Cu}_{14.7} \mathrm{Ni}_{5.3} \mathrm{P}_{22.5}$ nanowire at $250{ }^{\circ} \mathrm{C}$.

A short time per frame is another requirement needed to be satisfied in ECM experiment. Figure 7 shows $\tau$ measured on $\mathrm{Pt}_{57.5} \mathrm{Cu}_{14.7} \mathrm{Ni}_{5.3} \mathrm{P}_{22.5}$ nanowire at $250{ }^{\circ} \mathrm{C}$ using the $\mathrm{CCD}$ camera as a function of time per frame. All measurements have identical length of the time series (300s). $\tau$ has only small fluctuations with time per frame $\leq 0.1 \tau$, and the results are comparable to the results obtained with the $\mathrm{K} 2$ detector shown in Figure 2. With time per frame $>0.1 \tau$, the discrepancy from true relaxation time and the strong dependence of $\tau$ on the time per frame prove that the number of nanodiffraction patterns is insufficient to capture one relaxation time unit. Therefore, we conclude that the time per frame must be less than $0.1 \tau$ to guarantee sufficient sampling to obtain unreliable results. If $\tau$ is shorter than $1 \mathrm{~s}$, such as in the high temperature regime of $\mathrm{Pt}_{57.5} \mathrm{Cu}_{14.7} \mathrm{Ni}_{5.3} \mathrm{P}_{22.5}$ nanowire in Figure 2, the conventional slow CCD camera cannot be used and a fast direct electron detector is necessary. For the slower dynamics of the $\mathrm{Pd}_{40} \mathrm{Ni}_{40} \mathrm{P}_{20}$ system, $\mathrm{CCD}$ data are sufficient. Unlike the too-short time series, there is not a clear indicator in $g_{2}(t)$ for a too-slow camera, although there is some indication that $g_{2}(t)$ will show less of a flat plateau at short times than in Figure 1(b), and $\beta$ may become anomalously low.

\section{Conclusion}

We report first results using a fast direct electron detection camera for electron correlation microscopy experiments to measure the relaxation time of metallic glass alloys in their supercooled liquid state. We have applied ECM to investigate the supercooled liquid region of $\mathrm{Pt}_{57.5} \mathrm{Cu}_{14.7} \mathrm{Ni}_{5.3} \mathrm{P}_{22.5}$ glassy nanowire and $\mathrm{Pd}_{40} \mathrm{Ni}_{40} \mathrm{P}_{20} \mathrm{BMG}$ using fast $\mathrm{K} 2$ detector and CCD camera respectively. For $\mathrm{Pt}_{57.5} \mathrm{Cu}_{14.7} \mathrm{Ni}_{5.3} \mathrm{P}_{22.5}$, both $\tau$ and $\beta$ decrease over the whole measured supercooled liquid regime. For $\mathrm{Pd}_{40} \mathrm{Ni}_{40} \mathrm{P}_{20}$, the measured $\tau$ continuously decreases 
when temperature increases from $T_{g}+28{ }^{\circ} \mathrm{C}$ to $T_{g}+48{ }^{\circ} \mathrm{C}$ in the supercooled liquid and then slightly increases due to the onset of nucleation and crystallization. $\beta$ is quite scattered without tendency as temperature changes. ECM technique significantly improves the spatial resolution of relaxation dynamics measurement down to the nanometer scale compared with macroscopic experiments, but it requires a length of time series at least 40 times the characteristic relaxation time and a time per diffraction pattern at most 0.1 times the relaxation time. These requirements impose limitations on the accessible time scales imposed by sample drift at long times and detector speed at short times.

\section{Acknowledgement}

This research was supported by the US National Science Foundation under Contract No. DMR1506564 (PZ and PMV). The facilities and instrumentation for TEM sample preparation and microscopy at UW-Madison were supported by the University of Wisconsin Materials Research Science and Engineering Center (DMR-1121288), and access to the K2-IS camera was provided by the Center for Functional Nanomaterials, which is a U.S. DOE Office of Science Facility, at Brookhaven National Laboratory under Contract No. DE-SC0012704. Data storage and compression was carried out with support from the Center for Data Driven Discovery (C3D), part of the Computational Science Initiative (CSI) at Brookhaven National Laboratory https://www.bnl.gov/compsci/C3D/. $\mathrm{Pd}_{40} \mathrm{Ni}_{40} \mathrm{P}_{20} \mathrm{BMG}$ synthesis was supported by the U.S. DOE, Office of Basic Energy Sciences, Materials Science and Engineering Division, through the Ames Laboratory, Iowa State University (MFB and MJK). $\mathrm{Pt}_{57.5} \mathrm{Cu}_{14.7} \mathrm{Ni}_{5.3} \mathrm{P}_{22.5}$ nanorod synthesis was supported by NSF under Grant No. MRSEC DMR 1119826 (CRISP).

\section{References}

[1] L. He, P. Zhang, M.F. Besser, M.J. Kramer, P.M. Voyles, Electron Correlation Microscopy: A New Technique for Studying Local Atom Dynamics Applied to a Supercooled Liquid, Microsc. Microanal. 21 (2015) 1026-1033.

[2] P.N. Pusey, Intensity fluctuation spectroscopy of charged Brownian particles: the coherent scattering function, J. Phys. A. Math. Gen. 11 (1978) 119-135.

[3] M. Sutton, S. Mochrie, T. Greytak, S. Nagler, L. Berman, G. Held, et al., Observation of speckle by diffraction with coherent X-rays, Nature. 352 (1991) 608-610.

[4] A. Papagiannopoulos, T.A. Waigh, A. Fluerasu, C. Fernyhough, A. Madsen, Microrheology of polymeric solutions using x-ray photon correlation spectroscopy, J. Phys. Condens. Matter. 17 (2005) L279-L285.

[5] S.B. Dierker, R. Pindak, R.M. Fleming, I.K. Robinson, L. Berman, X-Ray Photon Correlation Spectroscopy Study of Brownian Motion of Gold Colloids in Glycerol, Phys. Rev. Lett. 75 (1995) 449-452.

[6] G. Grübel, F. Zontone, Correlation spectroscopy with coherent X-rays, J. Alloys Compd. 362 (2004) 3-11.

[7] W. Roseker, H. Franz, H. Schulte-Schrepping, A. Ehnes, O. Leupold, F. Zontone, et al., Development of a hard X-ray delay line for X-ray photon correlation spectroscopy and jitter-free pump-probe experiments at X-ray free-electron laser sources, J. Synchrotron Radiat. 18 (2011) 
$481-491$.

[8] H. Guo, G. Bourret, M.K. Corbierre, S. Rucareanu, R.B. Lennox, K. Laaziri, et al., Nanoparticle Motion within Glassy Polymer Melts, Phys. Rev. Lett. 102 (2009) 075702.

[9] M. Leitner, B. Sepiol, L.-M. Stadler, B. Pfau, G. Vogl, Atomic diffusion studied with coherent Xrays, Nat. Mater. 8 (2009) 717-720.

[10] O.G. Shpyrko, E.D. Isaacs, J.M. Logan, Y. Feng, G. Aeppli, R. Jaramillo, et al., Direct measurement of antiferromagnetic domain fluctuations, Nature. 447 (2007) 68-71.

[11] R.Z. Tai, K. Namikawa, M. Kishimoto, M. Tanaka, K. Sukegawa, N. Hasegawa, et al., Picosecond Snapshot of the Speckles from Ferroelectric $\mathrm{BaTiO}_{3}$ by Means of X-Ray Lasers, Phys. Rev. Lett. 89 (2002) 257602.

[12] J.M. Zuo, M. Gao, J. Tao, B. Li, R.D. Twesten, I. Petrov, Coherent nano-area electron diffraction, Microsc. Res. Tech. 64 (2004) 347-355.

[13] F. Yi, P. Tiemeijer, P.M. Voyles, Flexible formation of coherent probes on an aberration-corrected STEM with three condensers, J. Electron Microsc. (Tokyo). 59 Suppl 1 (2010) S15-21.

[14] P.G. Debenedetti, F.H. Stillinger, Supercooled liquids and the glass transition, Nature. 410 (2001) 259-267.

[15] H.B. Yu, W.H. Wang, K. Samwer, The $\beta$ relaxation in metallic glasses: an overview, Mater. Today. 16 (2013) 183-191.

[16] H. Wagner, R. Richert, Dielectric beta relaxations in the glassy state of salol?, J. Chem. Phys. 110 (1999) 11660.

[17] P. Wen, D.Q. Zhao, M.X. Pan, W.H. Wang, Y.P. Huang, M.L. Guo, Relaxation of metallic Zr ${ }_{46.75} \mathrm{Ti}_{8.25} \mathrm{Cu}_{7.5} \mathrm{Ni}_{10} \mathrm{Be}_{27.5}$ bulk glass-forming supercooled liquid, Appl. Phys. Lett. 84 (2004) 2790.

[18] J.C. Qiao, J.M. Pelletier, Dynamic Mechanical Relaxation in Bulk Metallic Glasses: A Review, J. Mater. Sci. Technol. 30 (2014) 523-545.

[19] J. Qiao, J.-M. Pelletier, R. Casalini, Correction to "Relaxation of Bulk Metallic Glasses Studied by Mechanical Spectroscopy,” J. Phys. Chem. B. 118 (2014) 648-648.

[20] G. Wilde, Slow relaxations in deeply undercooled metallic liquids, J. Non. Cryst. Solids. 312-314 (2002) 537-541.

[21] S. Kirsch, V. Frenz, W. Schärtl, E. Bartsch, H. Sillescu, Multispeckle autocorrelation spectroscopy and its application to the investigation of ultraslow dynamical processes, J. Chem. Phys. 104 (1996) $1758-1761$.

[22] G. Kumar, H.X. Tang, J. Schroers, Nanomoulding with amorphous metals, Nature. 457 (2009) 868-72.

[23] M.A. Van Huis, N.P. Young, G. Pandraud, J.F. Creemer, D. Vanmaekelbergh, A.I. Kirkland, et al., Atomic Imaging of Phase Transitions and Morphology Transformations in Nanocrystals, Adv. Mater. 21 (2009) 4992-4995.

[24] B. Ruta, Y. Chushkin, G. Monaco, L. Cipelletti, E. Pineda, P. Bruna, et al., Atomic-Scale Relaxation Dynamics and Aging in a Metallic Glass Probed by X-Ray Photon Correlation Spectroscopy, Phys. Rev. Lett. 109 (2012) 165701.

[25] R. Busch, E. Bakke, W.L. Johnson, Viscosity of the supercooled liquid and relaxation at the glass transition of the Zr46.75Ti8.25Cu7.5Ni10Be27.5 bulk metallic glass forming alloy, Acta Mater. 46 (1998) 4725-4732. 
[26] R. Busch, W. Liu, W.L. Johnson, Thermodynamics and kinetics of the $\mathrm{Mg}_{65} \mathrm{Cu}_{25} \mathrm{Y}_{10}$ bulk metallic glass forming liquid, J. Appl. Phys. 83 (1998) 4134.

[27] K. Schroter, G. Wilde, R. Willnecker, M. Weiss, K. Samwer, E. Donth, Shear modulus and compliance in the range of the dynamic glass transition for metallic glasses, Eur. Phys. J. B. 5 (1998) 1-5.

[28] L.M. Wang, R. Liu, W.H. Wang, Relaxation time dispersions in glass forming metallic liquids and glasses, J. Chem. Phys. 128 (2008) 5-10.

[29] C.Y. Lu, D.A. Vanden Bout, Effect of finite trajectory length on the correlation function analysis of single molecule data, J. Chem. Phys. 125 (2006) 124701. 